\title{
A Review of the Grasp Planning of Dexterous Hand
}

\author{
Wentao Cheng ${ }^{1, a^{*}}$, Ying Sun ${ }^{1,2, b}$ and Jiabing $\mathrm{Hu}^{2, \mathrm{c}}$ \\ ${ }^{1}$ Key Laboratory of Metallurgical Equipment and Control Technology, Ministry of Education, Wuhan \\ University of Science and Technology, Wuhan 430081, China. \\ ${ }^{2}$ Hubei Key Laboratory of Mechanical Transmission and Manufacturing Engineering, Wuhan \\ University of Science and Technology, Wuhan 430081, China. \\ a154072471@qq.com, bsunying65@wust.edu.cn, '461303771@qq.com \\ * The Corresponding author
}

Keywords: Dexterous hand; Grasp planning; Analysis of indexes; Problems and countermeasures

\begin{abstract}
With the continuous development of bionic machinery, dexterous hand development is also rising. And dexterous hands to complete a similar operation of the staff must be carried out planning. Firstly, the grasping of dexterous hand is classified. Then, four grasping analysis indexes of force closure, dexterity, static balance and stability are put forward according to classification. In the light of these indexes, the existing grasping planning method is analyzed on the basis of these indexes. Finally, the existing problems of the grasp planning method and the countermeasures are put forward.
\end{abstract}

\section{Introduction}

With the continuous development of science and technology and emerging interdisciplinary emerging, such as space exploration, medical equipment, nuclear energy development and so on have put forward higher requirements on the robot technology. The traditional industrial robot end gripper is designed for specific tasks and specific clamping parts. It has some limitations, such as poor flexibility and limited clamping mode. It has the characteristics of multi-degree of freedom and easy operation because the multi-fingered dexterous hands as a robot end of the characteristics of the actuator. In different situations, the object of various shapes can be crawled or dexterous. In recent decades, due to the urgent needs of engineering applications, the study of many dexterous hands has been developed rapidly. It has gradually become a specialized field of research, covering some important issues in the field of space exploration and medical devices [1]. Therefore, the research of dexterous hand is very important, both in theory and in engineering.

Manpower has a strong ability to adapt to grasp, which the main reason is because the people have the other four fingers relative to the thumb structure, and one can grasp in with the fingers movements of the palm. Such as the ability of physiological structure can change shape structure caught and adjust the preshape. The dexterity of robot dexterous hand is much lower than that of hand, so it is very important to grasp it.

\section{Multi-Fingered Dexterous Hand Grasping Classification}

Dexterous hands perform hand like movements, or grasp and manipulate target objects, and they must crawl. The core problem of grasp planning is how to select the position of the finger and palm relative to the object, that is to grasp the shape of the object. It mainly includes how to judge the stability, the selection of the position of the grab and the allocation of the grasping force, etc. People usually use two methods to study this problem: empirical method and analytic method. The guiding principle of empirical method is to simulate and study the process of grasping and manipulating objects by hand. The grasping experience of hand is applied to the grasp planning of dexterous hand. Among them, the most representative is Cutkosky in the absorption of previous research results based on the Grab Taxonomy [2], as shown in Fig. 1. 


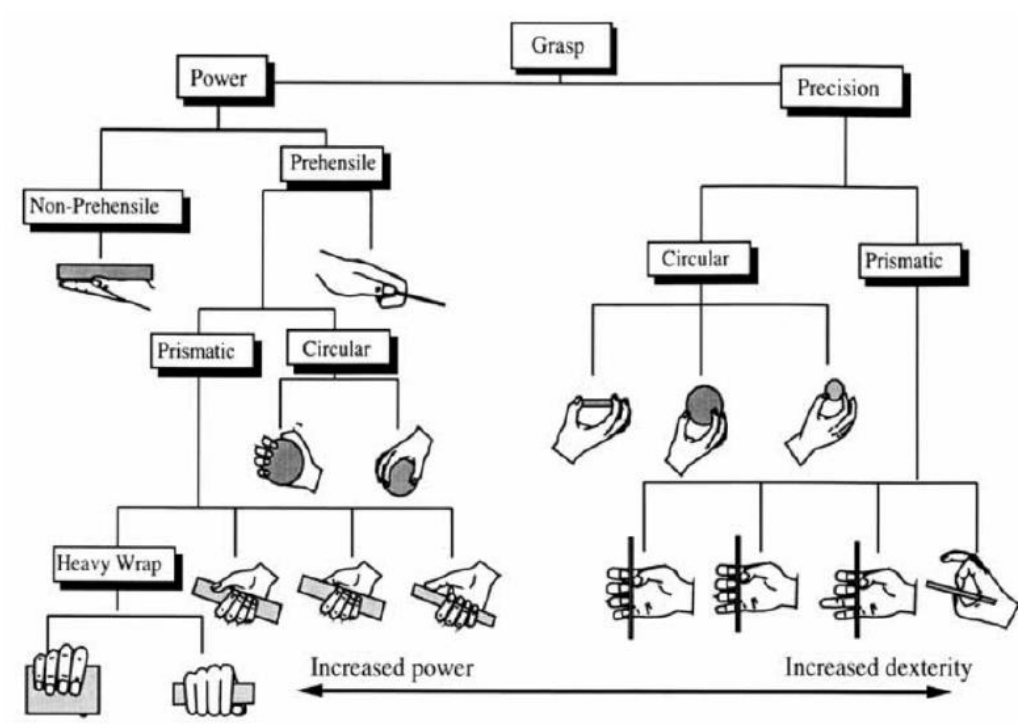

Figure 1. Finite Cutkosky grab sorting

Since the dexterous hand cannot achieve the complete imitation of human nature at present, the empirical method can only provide limited guidance, such as how to select the appropriate crawl mode, such as. Analysis of the guiding ideology of law is firstly established and dexterous hand grasping the accurate mathematical model, and then determine the contact constraints of kinematics and dynamics and other related models to describe the mathematical model of the entire collection system [3]. On this basis, some performance indexes are taken as the objective function, and the optimization method is used to realize the dexterous hand grasping planning. Analytical methods have been extensively and intensively studied.

\section{Grasp Planning Analysis Index}

Through the above analysis, analysis method for multi-finger dexterous hand grasping the role of some strong, detailed discussion of the following. The researchers usually carry out the analysis of multi-finger grasping from the following aspects: (1) Force closure; (2) Dexterity; (3) Static balance; (4) Stability.

Mason and Salisbury proposed the concepts of force closure and shape closure, and gave the conditions of guaranteeing force closure and shape closed grasping. When the dexterous hand grasping, grasping force against any known force closure grasping, special shaped closed grab belongs to force closure grasp, corresponding to all the contact point contact situation is smooth. Many researches focus on how to plan the contact points on the object to satisfy the force closed characteristics of grasping

Dexterity is mainly embodied in the following aspects: whether a grasping configuration can avoid singularity; whether it can obtain the maximum force or velocity in the desired direction; and whether each joint can keep away from the rotation boundary. Accordingly, researchers have proposed various reactions grasping dexterity index, such as grasping matrix the operability of the ellipsoid, the condition number, the smallest singular value, joint angle, joint deviation of the required torque, task ellipsoid, mission adaptability and dynamic operation.

Grasping static balance refers to the resultant force of a finger acting on an object. The screw is balanced with the external force of the object. It is a basic feature of the stability of grasping under static or quasi static conditions. The key problem is to obtain the optimal fingertip contact force under the condition of satisfying the relevant constraints. Kerr and Roth model the static load balancing problem into a linear programming model, and put forward the concepts of operation force and grasping internal force. The operation force is the force and the role of the object of phase equilibrium or overcome the external constraints of objects and change its motion and force of the role is to increase internal contact constraint, the dexterous hand can stably grasping objects so as 
not to fall, but the force is zero, that is to change the motion of the object does not produce effect [4,5]. Holzmann and Mc Carthy established the method of calculating the friction force under three finger grasping static balance. Nguyen established $n$ elastic fingers to achieve the static balance calculation algorithm. Li and Sastry discuss the decision criteria for obtaining stable grasping static equilibrium.

Grasping stability is the basic guarantee for dexterous hand to perform grasping and dexterous operation. Jameson and Leifer define the stability of grasping as two meanings: object stability and contact stability [6]. The former refers to the ability to return to the static equilibrium position when the object position is disturbed. The latter refers to the ability to prevent contact slippage when the object is subjected to force disturbances. Researchers have put forward various stability indicators from different angles.

Hanafusa and Asada firstly studied the stability of multi-fingered grasping. For the controllable multi-fingered hand, the stability index of minimizing the elastic potential energy was put forward. Nguyen has systematically studied the stability of $\mathrm{N}$ elastic finger plane and space grasping for the first time [7]. It is pointed out that the grasping stiffness matrix must be positive definite to ensure that the grasping is quasi-static and stable. Jameson and Leifer proposed a method to predict the stability of a given crawl, the conclusion is that for a total unknown to meet the constraints of equality and inequality capture, there is always finite solution, then the crawl is stable. In the light of contact stability, and He Yongqiang respectively put forward different stability indexes according to contact cone model [8].

\section{Existing grasp Planning Method}

Using the above indexes, scholars have proposed various description methods for grasp planning. Most of them build objective functions and constraints from the established performance indexes, and optimize the grasp planning. The grasp plan of dexterous hand can be realized by analytic method, and the optimal crawl can be realized, which is helpful for different tasks. However, due to uncertainty and variability, some tasks are difficult to be expressed in an abstract mathematical model. Therefore, how to obtain the information needed for modeling is the key to the problem. Moreover, analysis methods are often computationally complex and computationally efficient, and are generally applicable only to off-line programming $[9,10]$.

An effective solution is to combine the empirical method with the analysis method for multi-finger grasp planning, and researchers have made some attempts. Kang and Ikeuchi teach human experience to dexterous hand grasp planning and dexterous manipulation by teaching. First, the grasping action of human hand is segmented according to the time sequence, and the sequence of grasping action sequences is abstracted. Finally, the motion mapping of two levels of hand to dexterous hand is completed. Fischer by wearing a data glove hand measuring the angle of each joint, to determine the relationship between joint space and Cartesian space hand fingertip position based on neural network motion mapping in hand and dexterous hand work space, and motion planning of DLR dexterous hand [11,12]. Lee studied the motion analysis of human hand in human-computer interaction, and used it for teleoperation planning of Utah/MIT dexterous hand. Griffin adopts the shared control technology to implement the on-line grasp planning and control of dexterous hand, and realizes the combination of empirical method and analytic method. At the Beijing University of Aeronautics and Astronautics, Harbin Institute of technology and other universities have also carried out the work grasping planning research in this area. For example, $\mathrm{Li}$ Jiting and adopt master-slave mode to plan the grasp of multi-fingered hand $[13,14]$. By manually determining the contact point and mapping the position between the hand and the multi-fingered hand, the dexterous hand is used as the evaluation index of the dexterous hand to grasp the shape of the hand, and the optimal grasping performance is obtained. Liu Jie studied the dexterous hand grasp planning based on data glove, and classified the various crawl patterns with different classifiers, and carried out related virtual grasping experiments $[15,16]$. 


\section{The Existing Problems and Solutions}

People have carried out many years of research on the factors related to finger grasping, however, the multi-fingered manipulator still does not walk out of the laboratory $[17,18,19]$. The immature method of multi-finger grasping planning is one of the main reasons. Because multi-fingered grasping is a very complex research field, relates to a contact type, friction, grasping, grasping stability, all conform to the objective characteristics of modeling, grasp quality modeling and other aspects, and these factors are intertwined with each other, completely solve these problems also requires researchers to make unremitting efforts [20]. The author believes that the following aspects must be solved first.

The Existing Multi-Finger Grasping Planning Method is Integrated. The multi-fingered grasping planning method is still not mature, but after two years of hard work, people from the anthropomorphic grasping, machine learning to geometric analysis in various levels of grasping planning are put forward corresponding methods, so these methods should be studied, so as to establish a unified theoretical basis, these methods will be solved at different levels the organic combination, through high-level methods to achieve real-time algorithm, using the method of lower layers to ensure the accuracy of the algorithm, to form a comprehensive grasp planning method, the method can achieve a particular type of object capture, reached the practical requirements.

Study the modeling method of grasping Attributes. Grasp quality and grasp related to different grasping attributes concern different grasping purpose, measure method of capturing the different attributes are not the same, these properties include stability and operability (force and torque can be imposed), dexterity and precision. The grasp planning is ultimately reflected in the finger on the contact point position on the surface of the object, so it is necessary to study the modeling method of grasping attributes, establish various attributes and grasping contact position and grasping grasping contact geometry relationship is between.

Determine the Position of the Contact Point for the Form Seal. This is the basic problem of multi-fingered grasping, and is also the basis of all kinds of grasping quality optimization methods. Study on the determination and evaluation method of the quality of people grab more fully, but grasp planning methods currently in the generality and efficiency of all problems, so must be further studied to grab mathematical problems implied, research conditions guarantee form closed position relative to the contact point of each finger is arranged of grasping. The establishment of formal relations that form closure grasping finger contact point, find out the contact point form closed to meet all conditions.

\section{References}

[1] G.Hirziger, B.Brunner, K.Landzettel, et al. Autonomous Robot, (2003) No.14, p.127.

[2] I. Yamano, T.Maeno: Proceedings of the 2005 IEEE International Conference on Robotics and Automation Barcelona(Barcelona, Spain, April, 2005), p.2684.

[3] M.T.Mason, J.K.Salisbury: Robot Hands and the Mechanics of Manipulation (MIT Press, Cambridge, USA, 1985), p.3.

[4] S.C.Jacobsen, J.E.Wood, D.F.Knutti, et al. The International Journal of Robotics Research,Vol. 3 (1984) No.3, p.21.

[5] C.S. Lovchik, M. A. Difler: Proceedings of the IEEE International Conference on Robotics and Automation(Detroit, Michigan, 1999), p.907.

[6] Shadow Robot Company: Proceedings of the IEEE World Automation Congress (2004), p.123.

[7] G. Hirzinger, M. Fischer, B. Brunner, et.al. The International Journal of Robotics Research, Vol. 18(1999) No. 1, p.1064.

[8] S.Haidacher, J.Butterfass, M. Fischer, et al: Proceedings of the 2003 International Conference on Robotics and Automation (2003), p.684.

[9] T. Mouri, H. Kawasaki, K. Yoshikawa,et al: Proceedings of the 2002 IEEE ICCA(2002), p.1288.

[10] Y. Zhang, Z. Han, H. Zhang, et al: Proceedings of the 2001 IEEE International Conference on Robotics \& Automation (2001), p.2517. 
[11] X. H. Gao, M. H. Jin, L. Jiang, et al: Proceeding of the 2003 IEEE International Conference on Robotics \& Automation (Taipei, 2003), p.3164.

[12] H. Liu, G. Hirziger. Journal of Xi An Jiao Tong University, Vol.37(2003), No.4, p.331. (In Chinese)

[13] G.Jin, B.T. Qiu, F. Han. Electronic Design Engineering, Vol.24(2016), No.24, p.102. (In Chinese)

[14] S.W. Fan, Y.W. Liu, M.H. Jin, et al. Journal of Harbin Engineering University, Vol.30(2009), No.2, p.171.

[15] J.P. Gazeau, S. Zeghloul, M. Arsicault, et al: Proceedings of the 2001 IEEE International Conference on Robotics \& Automation(Seoul, May, 21-26,2001), p.2642.

[16]B. Yang, P.C. Huang, Q.H, Yang, et al. Journal of Mechanical \& Electrical Engineering, Vol.33(2016), No.4, p.388.(In Chinese)

[17] Albu, Schaera, Otfc,H.Greg. The International Journal of Robotics Research,Vol.26(2007), No.1, p.5.

[18] OTYC, ALBU-SCHAERA, KUGIA, et a1. IEEE Transactions on Robotics, Vol.24(2008), No.2, p.416.

[19] Y. Chen, F.Y. Xia. Journal of Chongqing University of Arts and Sciences, Vol.35(2016), No.2, p.79.(In Chinese)

[20]P.F. Ji, Y.H. Liu, C.G. Sun. MECHANICAL ENGINEER,(2017), No.2, p.66. 